\title{
Effect of reinforcement volume fraction and orientation on a hybrid tissue engineered aortic heart valve with a tubular leaflet design
}

\author{
Scott E Stapleton ${ }^{1 *}$, Ricardo Moreira ${ }^{2}$, Stefan Jockenhoevel ${ }^{2}$, Petra Mela ${ }^{2}$ and Stefanie Reese ${ }^{3}$
}

\begin{tabular}{l}
\hline *Correspondence: \\
scott.stapleton@ \\
rwth-aachen.edu \\
${ }^{1}$ Department of Mechanical \\
Engineering, University \\
of Massachusetts Lowell, \\
Lowell, MA 01854, USA \\
Full list of author information \\
is available at the end of the \\
article
\end{tabular}

${ }^{*}$ Correspondence: scott.stapleton@

rwth-aachen.edu

${ }^{1}$ Department of Mechanical

Engineering, University

of Massachusetts Lowell

Full list of author information article

\begin{abstract}
Transcatheter aortic valve implantation of fibrin-based tissue engineered heart valves with a tubular leaflet construct have been developed as an alternative to invasive traditional surgical heart valve implantation. In general, they are well suited for the pulmonary position, but display insufficient mechanical properties for the aortic position. To enable the application of tissue-engineered valves in the systemic circulation, the tissue is reinforced with a textile scaffold. The current study seeks to compare the effect of varying the fiber volume fraction and orientation of bidirectional textile reinforcement on the closed-valve configuration. An anisotropic large deformation material model based on structural tensors was chosen and the materials were characterized. A finite element model was constructed of the heart valve, and the pinching and suturing of the corners along with application of pressure was simulated. Virtual experiments were conducted with fiber volume fractions of $0.1,0.01,0.001$, and 0.0001 for $\pm 45^{\circ}$ fiber orientations. Furthermore, volume fraction was held at 0.01 and fiber orientations of $0^{\circ}$, $\pm 15^{\circ}, \pm 30^{\circ}, \pm 45^{\circ}, \pm 60^{\circ}, \pm 75^{\circ}$ and $90^{\circ}$ from the tube's axial direction were simulated and compared. It was shown that increasing the fiber volume fraction decreased the maximum principle strain in the valve, but lead to less closure. Additionally, the effect of fiber orientation affected the strains differently at different locations, depending on the local deformed geometry. This indicates that a non-uniform fiber distribution using tailored fiber placement could be used to optimize reinforcement design.
\end{abstract}

Keywords: Cardiovascular tissue engineering, Heart valve, Anisotropic elasticity, Large deformations, Textile reinforced composite, Finite elements

\section{Background}

Valvular heart disease is a steadily increasing socioeconomic burden worldwide. The proportion of valvular surgeries has increased over the past decades and these procedures now account for more than $20 \%$ of all cardiac surgeries with the number of replacement per year expected to triple by 2050 [1]. Tissue engineering (TE) of heart valves might overcome the well-known complications of contemporary devices implanted in young and elderly patients such as the need for a life-long anticoagulation therapy in the case of mechanical prostheses [2,3], the degeneration and therefore limited durability of the biological heart valves [2, 3] and the limited availability of homografts [4]. Furthermore, none of the available prostheses is capable of growth and remodeling. The basic concept

(c) 2015 Stapleton et al. This article is distributed under the terms of the Creative Commons Attribution 4.0 International License (http://creativecommons.org/licenses/by/4.0/), which permits unrestricted use, distribution, and reproduction in any medium, provided you give appropriate credit to the original author(s) and the source, provide a link to the Creative Commons license, and indicate if changes were made. 
of cell-based tissue engineering is to isolate/differentiate and expand tissue-specific cells derived from the patient and to seed them onto suitable matrix scaffolds. Subsequently, following in vitro cultivation and tissue development, the resulting structure is implanted into the recipient's organism. The ultimate goal is to provide the patient with an implant produced with and by the patient's own cells, able to remodel and self-repair, with physiological haemocompatibility, biologically and mechanically equivalent to the native tissue. Historically, TE heart valves have been designed to mimic the complex shape of the native valve in the attempt to recreate the natural haemodynamics [5].

Recently, we introduced a simplified design as an alternative to the classical one: a simple tissue-engineered tubular construct is sutured orthotopically in the aortic or pulmonary root at three sinotubular commissural points, and along a circumferential line at the annulus [6]. The tubular construct collapses inwardly under diastolic back pressure, closing the valvular conduit and opens again during systole (Fig. 1). The tubular leaflet valve features a low transvalvular pressure drop and a large effective orifice area. The design does not rely on any rigid support or sewing ring and can, therefore, be combined with a self-expanding stent for minimally invasive transcatheter valve implantation [7]. This procedure offers hope for patients who are considered inoperable or at high mortality risk with surgical implantation [7].

Since experimental testing of tissue-engineered heart valves can be extremely timeconsuming and expensive, numerical models are desired to predict the effect of geometrical and material changes on performance. One research group has created a series of models to simulate the closing of fiber-reinforced leaflets [8] and stentless fiber-reinforced heart valves [9] using high-performance polyethelyne fibers (HP-PE) in an ethylene-propylene-diene-monomer rubber matrix. These models utilized a continuum rule of mixtures technique to represent the fiber-reinforced rubber material only in elements which contain a fiber. As far as the authors can tell, the formulation did not track the angle change of fibers with loading except through rigid body rotations of the composite elements. Fiber orientation and volume fraction were investigated, and optimization techniques were applied to design the leaflets. Xiong et al. [10] compared a tubular and a molded geometry for stentless pericardial bioprostheses. An isotropic material model was utilized because the geometry was the focus of the study. More recently, Marom et al. [11] created a model with fluid-structure interaction (FSI) to determine the correlation

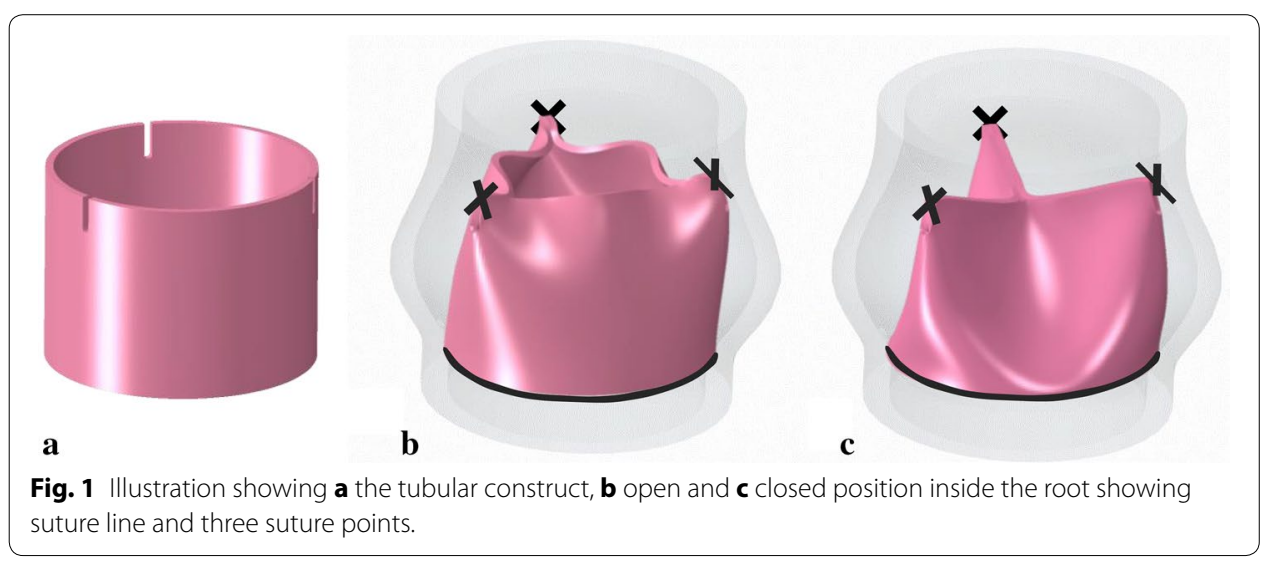


between root geometries and coaptation height and stresses. The effect of valve-specific collagen networks were also investigated [12] by mapping experimentally obtained collagen content and direction to an finite element (FE) model where collagen fibers were represented with beam elements. Cox et al. [13] created a simple, preliminary, FE model of the tubular heart valve geometry. While all of these modes represent varying degrees of modelling fidelity with aspects similar to the current study, none of them look at the effect of fiber reinforcement orientation for a tubular, tissue-engineered heart valve.

There are many different material models developed for modelling soft, biological materials reinforced by multiple fiber directions (for example $[14,15]$ and the reader is directed to [16] for a more exhaustive study). The model chosen for this particular study is based on the model first proposed by Reese et al. [17] and further developed and/or applied in [18-20]. The fiber directions are captured through so-called structural tensors, and the matrix is represented by a hyperelastic Neo-Hookean model. To compare the effect of different fiber volume fractions and orientations on the closing of the heart valve, an FE model was created to simulate the pinching/stitching and closing of the valve. Several fiber volume fractions and orientations were compared and improvements to the model were suggested.

\section{Methods}

In order to show the effect of fiber reinforcement volume fraction and orientation on a closed tubular heart valve, a finite element (FE) model was utilized. This section describes first the structural tensor material model adapted from Reese et al. [17] to describe the fiber-reinforced tissue under large deformation. Next, the material characterization is briefly summarized, and finally the boundary conditions of the model are defined.

\section{Material model formulation}

Consider an isotropic matrix material with $n_{f}$ fiber systems embedded within the matrix as shown in Fig. 2. The geometry of the fibers (diameter) is small compared to the global structure dimensions, such that the composite material can be viewed at the macro-scale and modelled as a homogenous continuum. Under traction and kinematic boundary conditions, a point within the material undergoes deformation and the right CauchyGreen tensor is defined in terms of the deformation gradient, $\mathbf{F}$, by

$$
\mathbf{C}=\mathbf{F}^{T} \mathbf{F}
$$



Fig. 2 Matrix material reinforced by $n_{f}$ fibers in the undeform med and deformed configurations. 
The fibers and matrix are assumed to perfectly bonded, which leads to the use of $\mathbf{C}$ for both fiber and matrix material models. The invariants of the intact strain tensor are used for the matrix, while only directional components are used for the fibers. The matrix model (superscript $m$ ) utilizes the first and third invariants of $\mathbf{C}$ :

$$
I_{1}^{m}=\operatorname{tr}(\mathbf{C}) \text { and } I_{3}^{m}=\operatorname{det}(\mathbf{C})
$$

while the model for fiber $i$ uses the first and second invariant of directional components of the strain:

$$
I_{1}^{f_{i}}=\operatorname{tr}\left(\mathbf{C M}_{f_{i}}\right) \text { and } I_{2}^{f_{i}}=\operatorname{tr}\left(\mathbf{C}^{2} \mathbf{M}_{f_{i}}\right)
$$

where the directional components of $\mathbf{C}$ are isolated using the structural tensor, $\mathbf{M}_{f i}$, defined as

$$
\mathbf{M}_{f_{i}}=\mathbf{N}_{f_{i}} \otimes \mathbf{N}_{f_{i}}
$$

and the vector $\mathbf{N}_{f_{i}}$ indicates the current direction of fiber $i$. The strain energy density of the material, $W$, is formulated as

$$
W=V_{m}\left(W_{N H}^{m}+W_{M L D}^{m}\right)+\sum_{i=1}^{n_{f}}\left(V_{f_{i}} W_{f_{i}}+\sum_{j=i+1}^{n_{f}} V_{f_{i j}} W_{f_{i j}}\right)
$$

where $V_{f_{i}}$ is the volume fraction of fiber $i$, and

$$
V_{m}=1-\sum_{i=1}^{n_{f}} V_{f_{i}} \text { and } V_{f_{i j}}=\frac{V_{f_{i}} V_{f_{j}}}{V_{f_{i}}+V_{f_{j}}} .
$$

Since the exact term for the coupling volume fraction, $V_{f_{i j}}$ is not yet determined, the equation presented here is built based on springs in series. If either of the two fibers has a 0 fiber volume fraction, the entire coupling term reduces to 0 . Determining a more exact equation for this term would be a topic for further research. The strain energy density of the matrix is represented by a Neo-Hookean contribution, $W_{N H}^{m}$ defined as

$$
W_{N H}^{m}=\frac{\mu}{2}\left(I_{1}^{m}-3\right)-\mu \ln \sqrt{I_{3}^{m}}+\frac{\lambda}{4}\left(I_{3}^{m}-1-2 \ln \sqrt{I_{3}^{m}}\right)
$$

where $\mu$ and $\lambda$ are the Lamé constants of the pure matrix material. Since it is well known that the Neo-Hookean model does not capture extreme stiffening for moderate and large deformations, an additional term for the isotropic matrix material, $W_{M L D}^{m}$ is included for medium/large deformations:

$$
W_{M L D}^{m}=K_{1}^{m}\left(I_{1}^{m}-3\right)^{\alpha_{1 m}}+K_{2}^{m}\left(I_{2}^{m}-3\right)^{\alpha_{2 m}}
$$

where $K_{1}^{m}, K_{2}^{m}, \alpha_{1 m}$, and $\alpha_{2 m}$ are constants attributed to the material properties of the matrix under moderate to large deformations. The strain energy density of the $i$ th fiber, $W_{f_{i}}$, is defined as

$$
W_{f_{i}}=K_{1}^{f_{i}}\left(I_{1}^{f_{i}}-1\right)^{\alpha_{1 f_{i}}}+K_{2}^{f_{i}}\left(I_{2}^{f_{i}}-1\right)^{\alpha_{2 f_{i}}}+K^{f_{i} m}\left(I_{1}^{m}-3\right)^{\alpha_{f_{i} m}}\left(I_{1}^{f_{i}}-1\right)^{\alpha_{f_{i} m}}
$$


where $K_{1}^{f_{i}}, K_{2}^{f_{i}}, \alpha_{1 f_{i}}$ and $\alpha_{2 f_{i}}$ are constants attributed to the material properties of the $i$ th fiber. The constants $K^{f_{i} m}$ and $\alpha_{f_{i} m}$ dictate how the matrix interacts with the $i$ th fiber. Furthermore, the coupling of the $i$ th and $j$ th fibers is captured by

$$
W_{f_{i j}}=K^{f_{i j}}\left(I_{1}^{f_{i}}-1\right)^{\alpha_{f_{i j}}}\left(I_{1}^{f_{j}}-1\right)^{\alpha_{f_{i f}}}
$$

where $K^{f_{i j}}$ and $\alpha_{f_{i j}}$ are constants dictating the fiber coupling. The second Piola-Kirchhoff stress tensor, $\mathbf{S}$, can be found by

$$
\mathbf{S}=2 \frac{\partial W}{\partial \mathbf{C}}
$$

which gives

$$
\mathbf{S}=V_{m}\left(\mathbf{S}_{N H}^{m}+\mathbf{S}_{M L D}^{m}\right)+\sum_{i=1}^{n_{f}}\left(V_{f_{i}} \mathbf{S}_{f_{i}}+\sum_{j=i+1}^{n_{f}} V_{f_{i j}} \mathbf{S}_{f_{i j}}\right)
$$

where

$$
\begin{aligned}
\mathbf{S}_{N H}^{m}= & \mu\left(1-\mathbf{C}^{-1}\right)+\frac{\lambda}{2}\left(I_{3}^{m}-1\right) \mathbf{C}^{-1}, \\
\mathbf{S}_{M L D}^{m}= & 2 \alpha_{1 m} K_{1}^{m}\left(I_{1}^{m}-3\right)^{\alpha_{1 m}-1} \mathbf{1}+2 \alpha_{2 m} K_{2}^{m}\left(I_{2}^{m}-3\right)^{\alpha_{2 m}-1}\left(I_{1}^{m} \mathbf{1}-\mathbf{C}\right), \\
\mathbf{S}_{f_{i}}= & 2 \alpha_{1 f_{i}} K_{1}^{f_{i}}\left(I_{1}^{f_{i}}-1\right)^{\alpha_{1 f_{i}}-1} \mathbf{M}_{f_{i}}+2 \alpha_{2 f_{i}} K_{2}^{f_{i}}\left(I_{2}^{f_{i}}-1\right)^{\alpha_{2 f_{i}}-1}\left(\mathbf{C} \cdot \mathbf{M}_{f_{i}}+\mathbf{M}_{f_{i}} \cdot \mathbf{C}\right) \\
& +2 \alpha_{f_{i} m} K^{f_{i} m}\left[\left(I_{1}^{m}-3\right)^{\alpha_{f_{i} m}-1}\left(I_{1}^{f_{i}}-1\right)^{\alpha_{f_{i} m}} \mathbf{1}+\left(I_{1}^{m}-3\right)^{\alpha_{f_{i} m}}\left(I_{1}^{f_{i}}-1\right)^{\alpha_{f_{i} m}-1} \mathbf{M}_{f_{i}}\right], \text { and } \\
\mathbf{S}_{f_{i j}=} & 2 \alpha_{f_{i j}} K^{f_{i j}}\left[\left(I_{1}^{f_{i}}-1\right)^{\alpha_{f_{i j}}-1}\left(I_{1}^{f_{j}}-1\right)^{\alpha_{f_{i f}}} \mathbf{M}_{f_{i}}+\left(I_{1}^{f_{i}}-1\right)^{\alpha_{f_{i j}}}\left(I_{1}^{f_{i}}-1\right)^{\alpha_{f_{i f}}-1} \mathbf{M}_{f_{j}}\right] .
\end{aligned}
$$

This phenomenological material model results in six unknown constants for the matrix, four per fiber, two for every pair of fibers for the fiber to fiber coupling, and two per fiber for the fiber to matrix coupling. The benefit of this general form with so many constants is that complicated textile structures with complex coupling between fibers and matrix can be represented, along with simple, aligned families of fibers. However, unless one uses virtual experiments to generate enough data to fit all of these constants, it may not be practical or possible to find values for each constant. For this study, the tissue matrix fails before $20 \%$ strain can be reached, which makes the additional term, $W_{M L D}^{m}$ to capture strongly non-linear behavior of the matrix unnecessary. Furthermore, the coupling between the fibers and the matrix is expected to have a relatively small influence on the overall response of the material, so $K^{f_{i} m}$ is assumed to be 0 . If the different fiber directions are not strongly coupled mechanically (for example through stitching or a tightly interwoven textile pattern like a warp-knit textile) then the coupled terms can be ignored and all $K^{f_{i j}}$ terms are considered to be 0 . Furthermore, since there is limited experimental data for the fibers, only the linear invariant term, $I_{1}^{f_{i}}$, is kept and $K_{2}^{f_{i}}$ is set to 0 . All of these simplifications result in a reduced second Piola-Kirchhoff stress tensor, which is: 


$$
\mathbf{S}=V_{m} \mathbf{S}_{N H}^{m}+\sum_{i=1}^{n_{f}}\left(V_{f_{i}} \mathbf{S}_{f_{i}}\right)
$$

where $\mathbf{S}_{N H}^{m}$ is as before, and

$$
\mathbf{S}_{f_{i}}=2 \alpha_{1 f_{i}} K_{1}^{f_{i}}\left(I_{1}^{f_{i}}-1\right)^{\alpha_{1 f_{i}}-1} \mathbf{M}_{f_{i}} .
$$

Furthermore, since the tissue matrix is much more compliant than the fibers, the fibers are expected to buckle in compression and therefore carry no axial compressive load. Hence,

$$
K_{1}^{f_{i}}= \begin{cases}0 & \text { if } f_{1}^{f_{i}}-1 \leq 0 \\ K_{1}^{f_{i}} & \text { if } f_{1}^{f_{i}}-1>0\end{cases}
$$

prevents the fibers from carrying any axial compressive loads.

\section{Material parameters}

To characterize the tissue, tensile tests were performed on heart valve tissue samples. The specimens had a gauge length of $10 \mathrm{~mm}$, a width of $5 \mathrm{~mm}$, and a nominal thickness of around $0.7 \mathrm{~mm}$. The specimens were preloaded with $0.005 \mathrm{~N}$, and stretched at $9 \mathrm{~mm} / \mathrm{min}$ until failure. The engineering stress vs. logarithmic strain is plotted in Fig. 3a. A one-element model with prescribed uniaxial displacement and unconstrained sides was used to fit the experimental data. The tissue was modelled as nearly incompressible and the Lamé constants, $\mu$ and $\lambda$, were found to be 0.57 and $5.12 \mathrm{MPa}$ respectively.

The fibers were chosen based on the textile reinforcement used in a heart valve by Weber et al. [6]. This study used polyethylene terephthalate (PET) fibers with $17 \mu \mathrm{m}$ diameter, 24 fibers per yarn, and the yarns had a spacing of approximately $1.6 \mathrm{~mm}$ (see Fig. 4). Although this valve had a warp-knit textile, the fibers for the current study were modelled as straight. The volume fraction of the fibers can therefore also be seen as a knock-down factor to account for the lack of straightness of the fibers within the textile. The fiber material parameters were obtained by using experimental data from Lechat et al. [21] as shown in Fig. 3b. This stress-strain curve is from a tensile test on a single
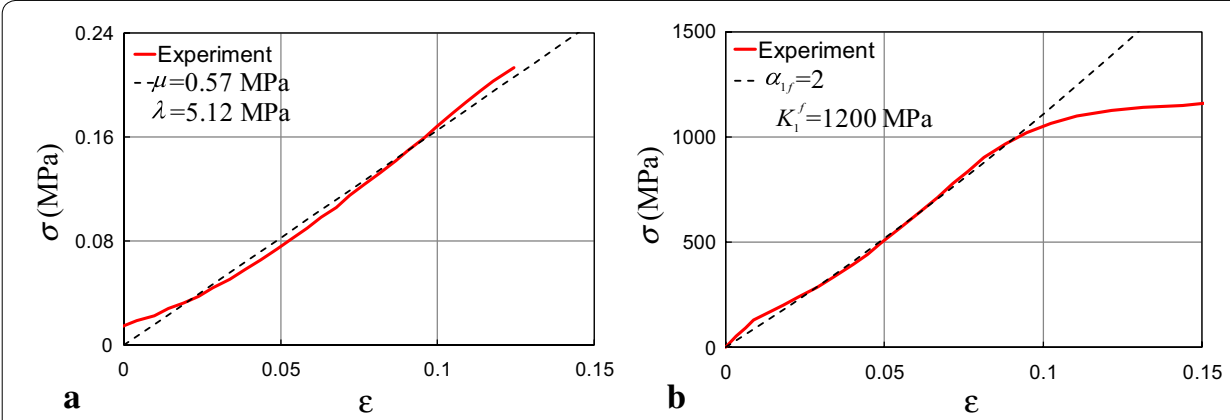

Fig. 3 Experimental uniaxial tensile data for the $\mathbf{a}$ engineered tissue and $\mathbf{b}$ PET fibers [21] used to fit the material constants. 




Fig. 4 Textile-reinforced tubular heart valve.

PET fiber. The same one-element model with unconstrained sides and one side given a prescribed displacement was used to fit the fiber parameters $K_{1}^{f_{i}}$ and $\alpha_{1 f_{i}}$ The data appeared to be linear initially (except for an initial non-linear period which is assumed by the authors to be remnants of the load transfer in the experimental setup) until it became plastic. Since plasticity was not included in the present model, the exponent $\alpha_{1 f_{i}}$ was assumed to be 2.0, making the stress-strain relationship for pure fiber linear in second Piola-Kirchhoff/right Cauchy-Green tensor space. $K_{1}^{f_{i}}$ was then found to be 1,200 MPa to fit the data for pure fiber in the fiber direction as shown in Fig. $3 \mathrm{~b}$.

\section{Finite element model}

The finite element (FE) model was created to simulate the suturing and closing of the heart valve and to show the effect of using different textile reinforcements. The FE model was built using the commercial software Abaqus, and an implicit integration solution scheme was used. The elements chosen were C3D8R elements (eight-noded brick elements with reduced integration and enhanced hourglass stiffness). The initial tube valve construct had a nominal diameter of $23 \mathrm{~mm}$, height of $18 \mathrm{~mm}$, and wall thickness of $0.7 \mathrm{~mm}$ as shown in Fig. 5.

To avoid excessive deformations and concentrated forces, the corner sutures were simulated using a control point-to-surface constraint. A $0.75 \times 2 \mathrm{~mm}$ square patch adjacent to the slit was coupled with a reference point located at the top midpoint of the patch (Fig. 5). The point was coupled with the translational degrees of freedom of the surface


Fig. 5 Geometric parameters of the undeformed valve model along with slit and loading patch details. 1/6th of the valve was modeled due to symmetry. 
(not rotational), and a continuum distribution using uniform weighting was used. Therefore the surface translations were tied to the control point, but only in an average sense. A similar control point and patch were placed in the center of the leaflet to force the valve closed, thereby bypassing snap-through of the leaflet. Utilizing a control point and load patch allowed applying boundary conditions to the control point without having the entire load applied at a concentrated point on the valve surface.

The boundary conditions and loading steps can be summarized as follows (see Fig. 6):

- Initial step: The bottom of the valve was fixed (encastre) and symmetry surfaces were given symmetric boundary conditions perpendicular to the surface. Contact was enforced between the inside valve surface and rigid wall.

- Step 1: The control points were given a prescribed a displacement in the $x$-direction $\left(U_{x}\right)$, bringing them against the contact plane.

- Step 2: The control point near the slit was fixed in space (simulating suture to stent) and a uniform pressure of $11 \mathrm{kPa}$ [9] was applied to the outer surface of the valve.

One of the main difficulties in an implicit simulation of a rather thin-walled, flexible tubular structure is bifurcation of solutions. In many instances during the simulation loading, there were negative eigenvalues indicating buckling or unstable solutions. The first and most obvious case was when initially suturing and pinching the tube. Initial models attempted to pinch the corners and then subsequently applying pressure. This caused snap-through of the tube wall, and convergence was extremely difficult. To avoid snap-through, the control point in the middle of the leaflet was introduced so that it was simultaneously closed and pinched (see Fig. 6).

Additionally, an initial full-valve model also revealed many different buckling modes that caused convergence difficulties. Some of these modes are shown in simplified drawings in Fig. 7. The modes shown in Fig. 7a-c all involve buckling of the closed lids. This can be extremely sensitive to the boundary conditions, and where the corner sutures are placed. The mode shown in Fig. 7d occurs when the leaflets first come into contact. One lip may slip above the other two and cause an asymmetrical configuration. Furthermore, another mode which the valve can display is shown in Fig. 7e, in which one leaflet forces its way in between the other two leaflets.

The presence of all of these alternate solutions can cause solution convergence difficulties for an implicit simulation. To force the valve into a symmetrical state and remove alternate asymmetric solutions, only $1 / 6$ th of the valve was modelled. To model

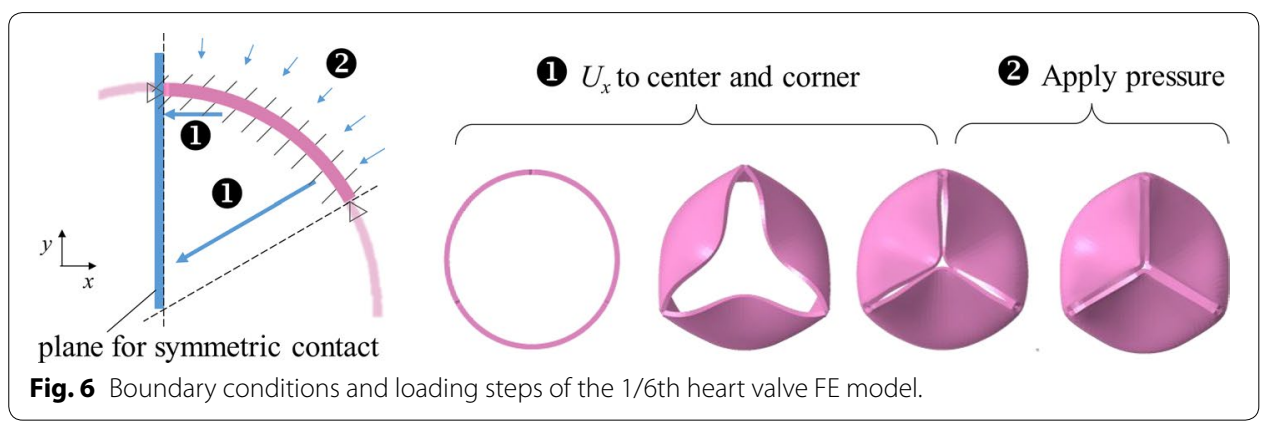






the symmetric contact of the valve leaflet with the adjacent leaflet, a contact plane was inserted into the model and soft contact was enforced between the plate and the inner surface of the valve wall as shown in Figs. 6 and 8a.

A $0.7 \mathrm{~mm} \times 3.35 \mathrm{~mm}$ slit was inserted on the three corners where a suture is placed to give the tube its valve structure (Fig. 8). While this was not necessarily representative of actual heart valves, it was necessary numerically to avoid elements with excessive distortion which make solution convergence difficult. A physical justification of this practice may lie in the conditioning process of actual heart valves [22]. The construct consisting of cell-laden fibrin and the textile reinforcement is pinched and sutured into place, and the cells grow and multiply in a bioreactor while the valve is opening and closing. During this opening and closing, the pinched corners with a suture are not opening and closing. Therefore, cells are growing in this region in the bent configuration. Therefore, the stresses in the pinched regions in the actual heart valve are unknown, but are probably much less than if the fully-grown material were pinched and sutured. These corner regions are not considered critical in the analysis and the slits are inserted to allow better solution stability.

Three studies were conducted: a study on element size convergence, fiber volume fraction, and fiber orientation. The various material and geometric parameters used each study are summarized in Table 1 . Prior to comparing heart valves with different fiber angle orientations, convergence with increasing mesh density was found for a heart valve with no fiber reinforcement. Elements were kept roughly square, and meshes were generated with one, two, three, and up to six elements through the thickness. Convergence

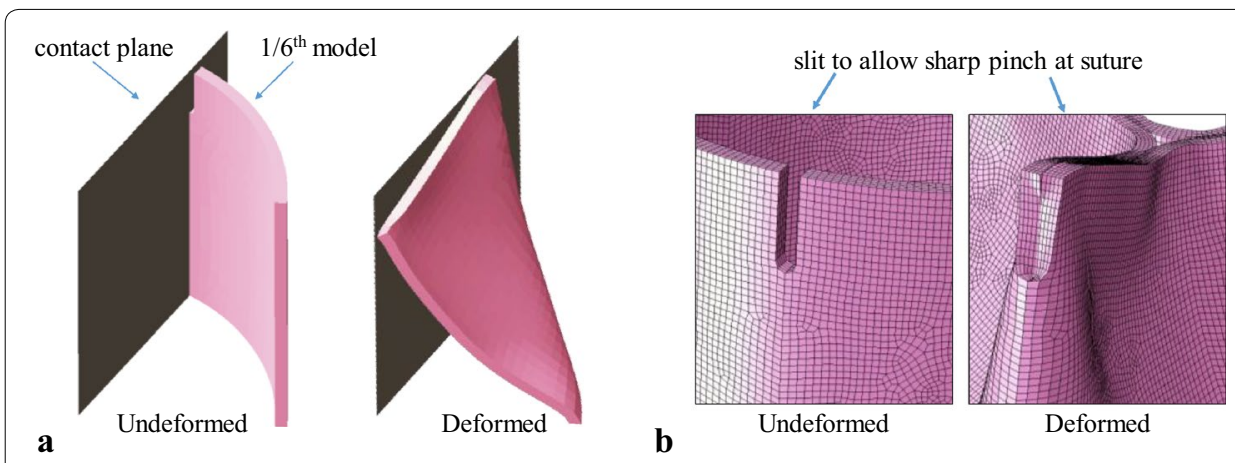

Fig. 8 Model details: a a rigid wall along symmetry line to enforce symmetric contact and $\mathbf{b}$ a slit at the pinched corner near the suture to avoid excessive element distortion. 
Table 1 Geometric and material parameters for the heart valve model

\begin{tabular}{|c|c|c|c|c|c|c|c|}
\hline Study & $\mu$ (MPa) & $\lambda(\mathrm{MPa})$ & $K_{1}^{\kappa}(\mathrm{MPa})$ & $\alpha_{f}$ & $\theta_{i}\left({ }^{\circ}\right)$ & $V_{f i}$ & $n_{e l}\left(\times 10^{3}\right)$ \\
\hline $\begin{array}{l}\text { Mesh size } \\
\text { convergence }\end{array}$ & & & & & NA & 0 & $\begin{array}{l}0.5,3,11,18,27 \\
100\end{array}$ \\
\hline Volume fraction & 0.55 & 275 & 1,200 & 2 & \pm 45 & $\begin{array}{r}0.01,0.001 \\
1 \times 10^{-4} \\
1 \times 10^{-5^{\prime}}\end{array}$ & 18 \\
\hline Fiber orientation & & & & & $\begin{array}{l}0, \pm 15, \pm 30, \pm 45 \\
\quad \pm 60, \pm 75,90\end{array}$ & 0.001 & 18 \\
\hline
\end{tabular}

was judged by looking at the average relative error of the maximum principle strain of the leaflet centerline. The average relative error, $\bar{\varepsilon}_{\text {error }}$, was defined as

$$
\bar{\varepsilon}_{\text {error }}=\frac{1}{n_{e l}} \sum_{n_{e l}}^{i=1}\left|\frac{\varepsilon_{p}^{c m}\left(x_{i}\right)-\varepsilon_{p}^{f m}\left(x_{i}\right)}{\varepsilon_{p}^{f m}\left(x_{i}\right)}\right|
$$

where $n_{e l}$ is the number of elements along the leaflet centerline as defined in Fig. 9b, $\varepsilon_{p}^{c m}\left(x_{i}\right)$ is the maximum principle strain evaluated at $x_{i}$ in the current mesh (mesh under consideration), $\varepsilon_{p}^{f m}\left(x_{i}\right)$ and is the maximum principle strain evaluated at $x_{i}$ in the finest mesh (six elements through the thickness). The heart valve model without fiber reinforcement results were loaded at a pressure of $6 \mathrm{kPa}$ rather than $11 \mathrm{kPa}$. This was because the tissue was so compliant that the entire valve collapsed above $6 \mathrm{kPa}$, making the results not comparable to the fiber reinforced valves and causing solution convergence difficulties.

Finally, the maximum principle strain of heart valves with different fiber volume fractions and orientations was determined. The mesh size used was five elements through the thickness and the remaining two directions roughly $0.23 \times 0.23 \mathrm{~mm}$. The results were recorded at $11 \mathrm{kPa}$ of applied pressure. The maximum principle strain contours are presented here and the strain along the leaflet centerline (Fig. 9b) and along the line under the corner stitch were shown because they contained the points of highest strain. However, the magnitudes near the bottom suture and the corner stitch should be considered suspect and useful for more of a qualitative rather than quantitative comparison. For the
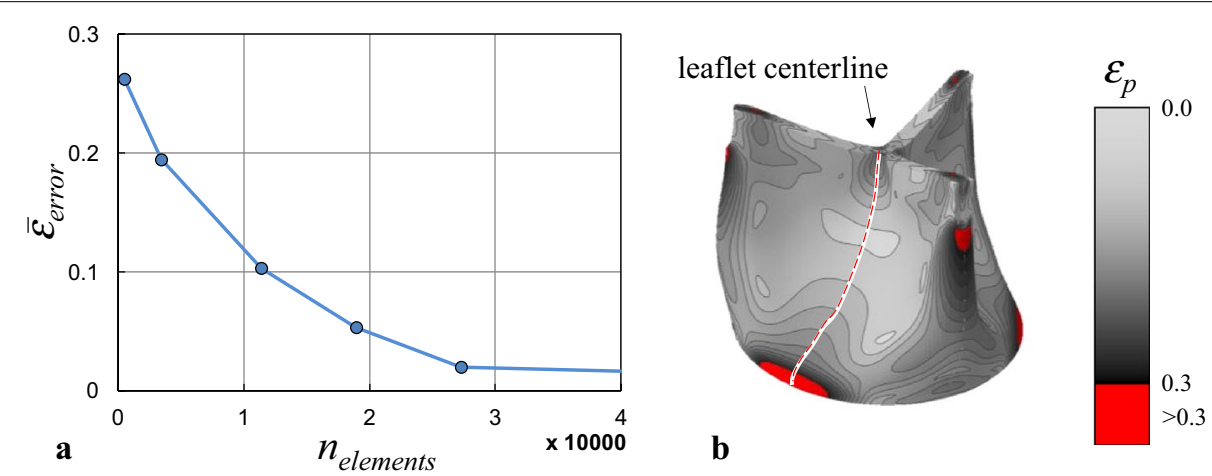

Fig. 9 Elemental convergence study for heart valve with no fiber reinforcement; $\mathbf{a}$ average error at the leaflet centerline and $\mathbf{b}$ maximum principle strain distribution in the valve. 
bottom suture, the bottom surface of the heart valve FE model is fixed. It is expected that a suture line would not be entirely fixed, but allowed some rotation. Therefore, the actual boundary probably lies somewhere between a fixed and a pinned condition. The corner stitch strains are also suspect, because the growth of the tissue takes place in the stitched configuration, which most likely leads to lower strains in the material around this point.

\section{Results and discussion}

To compare the effect of reinforcement fiber volume fraction and orientation on the closing of a tubular heart valve, the finite element (FE) model was created and utilized. First, the ability of the model to converge with decreasing mesh size was investigated for the heart valve model without textile reinforcement. Finally, the fiber volume fraction and fiber reinforcement orientation was investigated to show how the fiber reinforcement can be tailored to decrease maximum strain or change valve shape under pressure.

\section{Mesh size convergence}

First, the elemental convergence of the heart valve model was studied for a heart valve without fiber reinforcement. The average deviation of the maximum principle strain from the model with the finest mesh (6 elements through the thickness) was used to show elemental convergence.

The average deviation error is plotted in Fig. 9a for different meshes. As can be seen, the average deviation is quite low even for coarse meshes. The spot on the centerline along the bottom suture is the only location where mesh size convergence was not immediately achieved. Since the boundaries were not entirely representative of actual conditions as discussed earlier, the mesh size used was five elements through the thickness with the other dimensions roughly $0.23 \times 0.23 \mathrm{~mm}$, resulting in 18,943 elements.

\section{Effect of fiber volume fraction}

After convergence was shown, the heart valve FE model was run to the full pressure of $11 \mathrm{kPa}$ for $\pm 45^{\circ}$, fiber orientations and a fiber volume fraction of $0.01,0.001,0.0001$, and 0.00001 . Contour plots of the maximum principle logarithmic strain on the surface of the heart valves with different fiber volume fractions is plotted in Fig. 10. By looking at the strain distributions, there are aspects to be highlighted. First, the highest points of strain are located just below the slit in the valve corner. This region undergoes extreme bending and deformation, even with the slit. The maximum principle strain here surpasses the maximum strain in the specimen shown in Fig. 3a. As stated earlier, in an actual heart valve, the corner is stitched and then the cells are subsequently grown onto the fibrin/textile scaffolding. During growth, the valve is conditioned by pumping fluid and opening and closing the valve. However, since the stitched corner does not move during opening/closing, the cells grow on the corner without any residual stress. While it is true that the scaffolding will have some residual stress in the corner, the tissue probably will not. Therefore, the strain magnitude predicted here is expected to be higher than for the actual valve.

Another thing to note is that there is a strain peak on the valve side below the stitch. This point shows the influence of the corner control point on the valve lip. The control point is fixed while pressure is applied, so there is an increase of strain here due to 


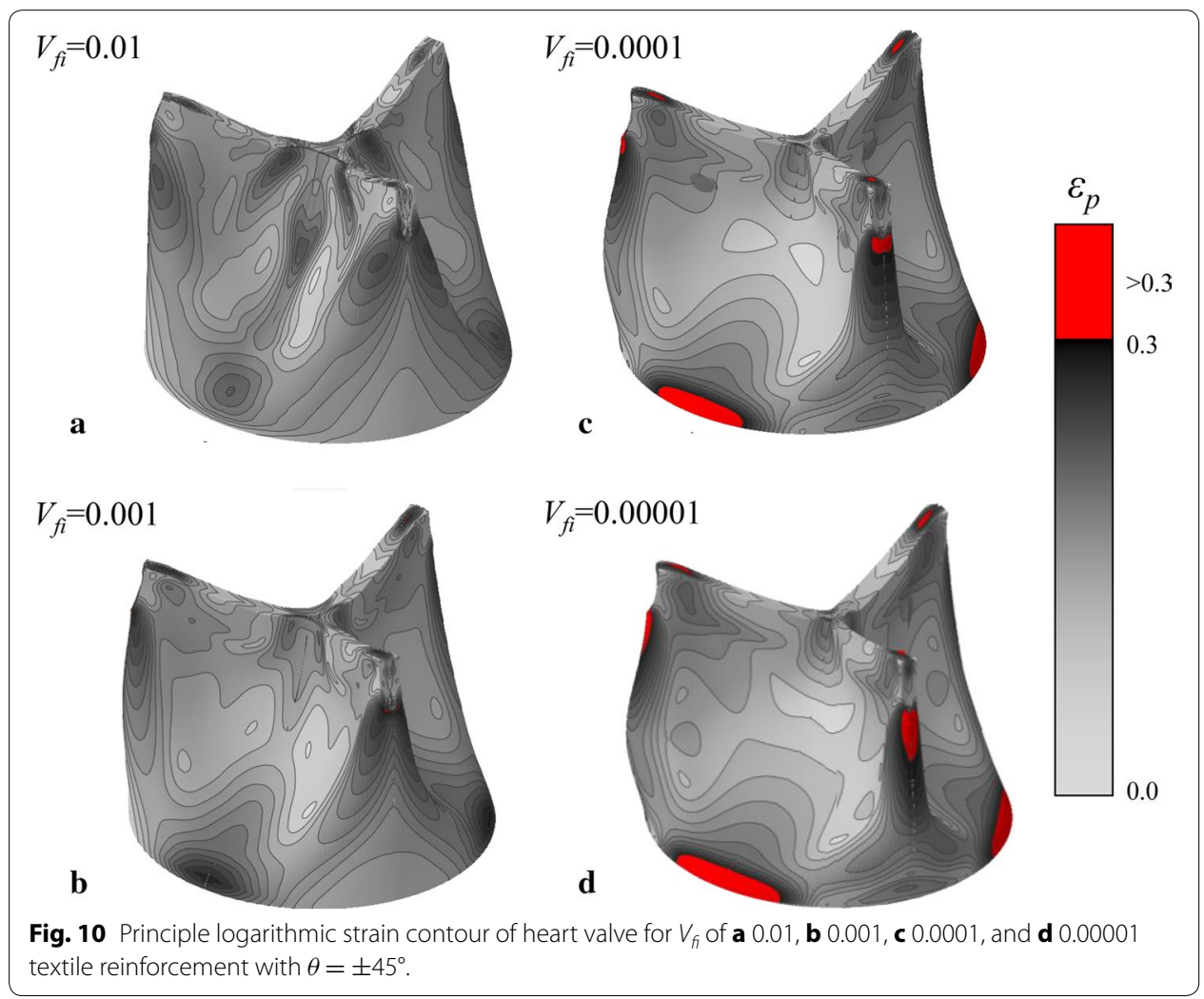

the control point keeping the valve up. If this stitch boundary condition were applied to a single point rather than through a control point with loading patch, a much higher strain peak would be seen along with excessive localized deformation. Other than the corners, the second highest strain is located at the base of the valve. Like the corner, strains higher than those allowed by the material are found here. It should be noted that the fixed boundary conditions at the base are probably stiffer than the actual valve. One row of sutures holds the valve to the stent, and the suture probably behaves somewhere in between a fixed and a pinned boundary.

A more detailed look at the principle logarithmic strains can be seen in Fig. 11 along the leaflet centerline (Fig. 11a) and the line below the corner suture (Fig. 11b). These regions were both chosen because they normally contained the maximum strains. As one would expect, higher volume fractions resulted in less bending of the valve and less strain, especially in the corner positions. Along the leaflet centerline in Fig. 11a, the stiffer valve with fiber volume fraction of 0.01 shows a more even distribution of strain because the valve does not collapse and fold at one single point as do the others.

The effect of fiber volume fraction is fairly intuitive. The higher the volume fraction, the stiffer the valve and the lower the strains are generally. Lower strains usually mean higher strength. On the other side, the stiffer the valve the less it closes. The valve with fiber volume fraction of 0.01 still has quite a bit of opening at the top, while the lower volume fractions are completely closed. Due to this tradeoff between strength and closure (or coaption), one needs to define (1) the minimum closure required for the valve to fulfill its function, (2) define the closure time required and then chose the highest 



Fig. 11 Principle logarithmic strain plot $\mathbf{a}$ below the corner stitch and $\mathbf{b}$ in the middle of the leaflet on the surface of the heart valve with different fiber volume fractions and $\pm 45^{\circ}$ fiber orientation.

volume fraction which fulfills both requirements. In this way, a balance can be found between strength and function.

\section{Effect of fiber orientation}

After isolating the effect of volume fraction on the maximum principle strains in the heart valve, the fiber orientation was investigated. The volume fraction per fiber direction was held constant at 0.001 , and fiber orientations of $0^{\circ} \pm 15^{\circ}, \pm 30^{\circ}, \pm 45^{\circ}, \pm 60^{\circ}$, $\pm 75^{\circ}$, and $90^{\circ}$ were compared. Contour plots of the maximum principle logarithmic strain on the surface of the heart valves with different fiber reinforcement orientations are plotted in Fig. 12. The maximum principle logarithmic strains plotted along critical lines are compared in Fig. 13. The two locations of stress concentrations: at the base of the valve along the leaflet centerline at near the pinch, show reductions in strain concentration intensity for different fiber orientations. For shallower angles such as 0,15 , and $30^{\circ}$, the pinched areas are is less-supported and the strains here are high. The steeper angles like 60,75 , and $90^{\circ}$ result in reduced strain near the base of the valve. The $45^{\circ}$ specimens appear to be a good balance between the two. However, to really minimize the strain in both locations without increasing fiber volume fraction, the orientation would need to be locally tailored. This highlights the fact that the most beneficial reinforcement orientation depends on the location in the valve, which makes a case for a locally optimized fiber orientation in the future (similar to [23-26]).

\section{Future work}

This model serves to compare the effects of fiber volume fraction and orientation on the closed-valve configuration, but there are many aspects of the model that could be improved to move towards a predictive rather than merely comparative model. First, more material data is required for proper modelling of the fiber-reinforced tissue. Some tests with the fiber-reinforced material would be necessary to not only validate the model but also to include more advanced aspects including fiber-matrix coupling and fiber-fiber coupling. This could be done by physical testing or by creating a unit-cell model of the textile in a tissue matrix and then conducting virtual experiments. Additionally, experimental data of a textile-reinforced heart valve would be necessary for a validation of the model. 


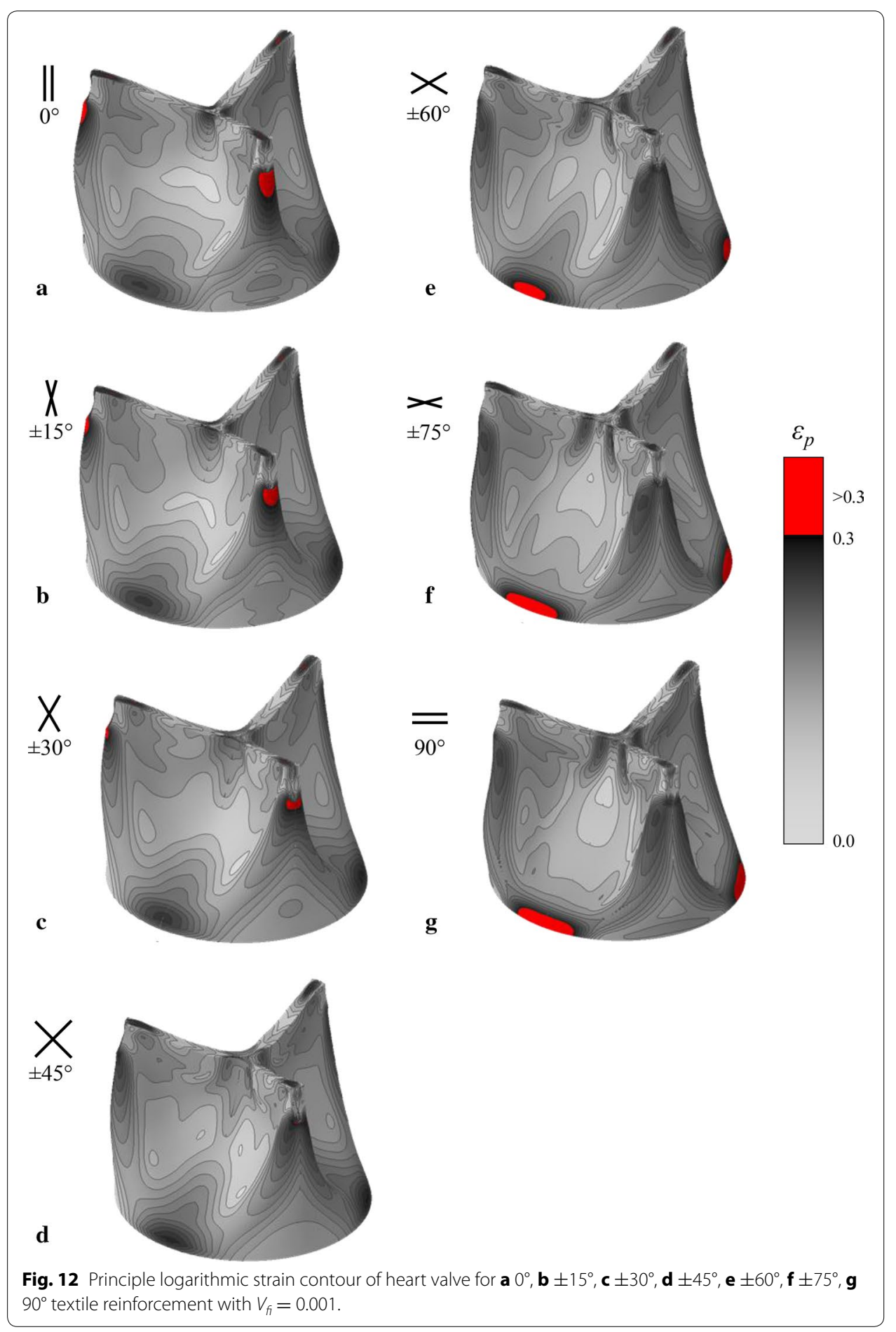

Furthermore, there could be some improvements on the material modelling. The material model treats the tissue with textile as a homogenous material. For membrane stresses, this is probably a reasonable approximation. However, since the soft tissue grows on the outer surface of the textile, the bending stiffness will be much lower in the actual material. Either a multi-layered continuum model or a shell element with bending stiffness based 

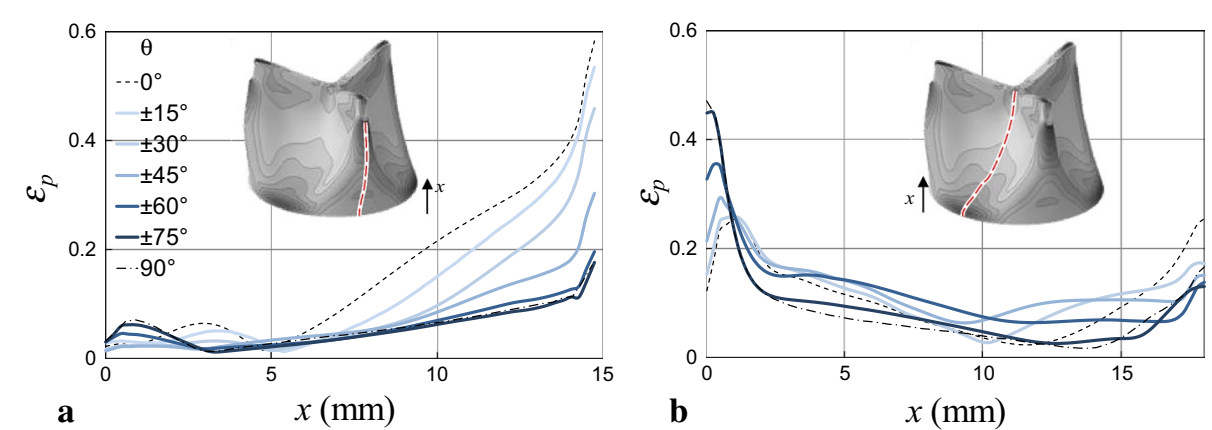

Fig. 13 Principle logarithmic strain plot $\mathbf{a}$ below the corner stitch and $\mathbf{b}$ in the middle of the leaflet on the surface of the heart valve with different reinforcement fiber orientations.

on textile location would be an improvement. The tissue is in actuality not an isotropic material but anisotropic due to conditioning and the fibrous collagen growth in the loading directions. This would mean that not only is the tissue anisotropic, but the material directions would be spatially varying. A more advanced material model could be implemented and a conditioning simulation could be carried out to obtain the local material anisotropy in the conditioned valve similar to [27, 28]. Additionally, plasticity of the tissue and fibers could be included to more accurately predict failure of the valve.

Finally, some FE improvements would lead to a more realistic description of the problem. Running a dynamic simulation with an explicit solver would help with solution instability and contact convergence, and be able to account for the dynamic pressure application in a real valve. A full model of the heart would include the non-symmetric modes, which may appear under more realistic boundary conditions. All of these improvements should be topics of future work to yield a higher-fidelity prediction for the behavior of textile-reinforced heart valves.

\section{Conclusion}

The goal of the current study was to show the effect of changing fiber reinforcement orientation on a textile reinforced tubular heart valve through virtual experimentation. First, a large deformation, anisotropic material model first introduced by Reese et al. [17] was adapted. This model is based on the concept of structural tensors, which isolates directional components of the right Cauchy-Green tensor and uses a corresponding invariant in the energy formulation. The matrix was modelled as a nearly incompressible Neo-Hookean isotropic material. The many of the coupled and higher order terms were neglected because a lack of sufficient experimental data would make the fitting of many constants non-singular and arbitrary.

A finite element (FE) model was constructed for the heart valve. To improve computational efficiency and avoid asymmetric buckling modes, one-sixth of the tube was modelled. Symmetry boundary conditions were applied on both sides and the bottom was fixed to represent the suture to the stent. This last boundary condition is probably stiffer than a suture would be in reality. Three slits were cut out of the tube where the tube would be pinched and sutured to avoid excessive distortion at one single point. This is justified by the fact that the cells grow in the pinched deformation, making the residual stresses at the corner smaller than represented in the model. The valve was closed and pinched 
simultaneously to avoid snap-through instability, then a uniform pressure was applied to the outside surface. A contact plane was placed in the middle for contact symmetry.

A few studies were carried out with the FE model. First, elemental convergence was shown by comparing the maximum principle strains of the leaflet centerline. Good convergence was achieved for relatively coarse meshes, but the solution around the stress concentrations required finer meshes. Second, the effect of fiber volume fraction was shown be comparing valves with $0.01,0.001,0.0001$, and 0.00001 fiber volume fractions for $\pm 45^{\circ}$ fiber orientations. An increase in fiber volume fraction reduced the maximum principle strain at critical locations because the valve was stiff enough that it did not fold at a concentrated location. However, higher volume fraction also resulted in less valve closure, illustrating the trade-off between strength and stiffness. A more optimum valve could be constructed by reinforcing critical areas with more fibers and leaving other areas with fewer fibers to achieve better closure. Finally, fiber reinforcement orientations of $0^{\circ}, \pm 15^{\circ}, \pm 30^{\circ}, \pm 45^{\circ}, \pm 60^{\circ}, \pm 75^{\circ}$, and $90^{\circ}$ were applied and maximum principle strain distributions were compared. It was shown that the fiber reinforcement stiffened the valve, but different orientations were beneficial at different locations. This suggests the use of fiber placement technology to optimize the fiber direction to increase the strength of a heart valve without adding a large amount of fibers.

\section{Authors' contributions}

SES performed the FE analysis and drafted most of the manuscript. RM performed the tissue tensile tests. SJ developed the textile reinforced heart valve concept. PM participated in the design and coordination of the study, wrote parts of the manuscript, and determined the boundary conditions for the valve. SR conceived much of the study, reviewed and edited the manuscript, and created the material model. All authors read and approved the final manuscript.

\section{Author details}

${ }^{1}$ Department of Mechanical Engineering, University of Massachusetts Lowell, Lowell, MA 01854, USA. ${ }^{2}$ Department of Tissue Engineering and Textile Implants, Institute of Applied Medical Engineering, RWTH Aachen University, Aachen 52074, Germany. ${ }^{3}$ Institute of Applied Mechanics, RWTH Aachen University, Aachen 52074, Germany.

\section{Acknowledgements \\ This research was funded by the Integrated Interdisciplinary Institute of Technology for Medicine (I $\left.{ }^{3} \mathrm{TM}\right)$ of RWTH Aachen University through Seed-Fund SF_14-4-08, "Hybrid scaffold for a transcatheter tissue-engineered aortic valve". Additional funding was provided by the People Programme (Marie Curie Actions) of the European Union's Seventh Framework Pro- gramme FP7/2007-2013/under REA Grant Agreement No 317512. The authors would additionally like to thank Bertram Stier, Daniel Höwer, Dennis Della Corte and Deepanschu Sodhani for their helpful discussion and support.}

Compliance with ethical guidelines

Competing interests

The authors declare that they have no competing interests.

Received: 25 March 2015 Accepted: 15 July 2015

Published online: 13 August 2015

\section{References}

1. lung B, Vahanian A (2011) Epidemiology of valvular heart disease in the adult. Nat Rev Cardiol 8:162-172. doi:10.1038/nrcardio.2010.202

2. Padala M, Keeling WB, Guyton RA, Thourani VH (2011) Innovations in therapies for heart valve disease. Circ J 75:1028-1041

3. Pibarot P, Dumesnil JG (2009) Prosthetic heart valves selection of the optimal prosthesis and long-term management. Circulation 119:1034-1048. doi:10.1161/CIRCULATIONAHA.108.778886

4. Llames S, García E, Otero Hernández J, Meana Á (2012) Tissue bioengineering and artificial organs. In: López-Larrea C, López-Vázquez A, Suárez-Álvarez B (eds) Stem cell transplantation. Springer, US, pp 314-336

5. Butcher JT, Mahler GJ, Hockaday LA (2011) Aortic valve disease and treatment: the need for naturally engineered solutions. Adv Drug Deliv Rev 63:242-268. doi:10.1016/j.addr.2011.01.008

6. Weber M, Heta E, Moreira R, Gesche VN, Schermer T, Frese J et al (2014) Tissue-engineered fibrin-based heart valve with a tubular leaflet design. Tissue Eng Part C Methods 20(4):265-275 
7. Moreira R, VelzT, Alves N, Gesche VN, Malischewski A, Schmitz-Rode T et al (2014) Tissue-engineered heart valve with a tubular leaflet design for minimally invasive transcatheter implantation. Tissue Eng Part C Methods. doi:10.1089/ten.TEC.2014.0214

8. De Hart J, Cacciola G, Schreurs PJ, Peters GW (1998) A three-dimensional analysis of a fibre-reinforced aortic valve prosthesis. J Biomech 31:629-638

9. Cacciola G, Peters GW, Schreurs PJ (2000) A three-dimensional mechanical analysis of a stentless fibre-reinforced aortic valve prosthesis. J Biomech 33:521-530

10. Xiong FL, Goetz WA, Chong CK, Chua YL, Pfeifer S, Wintermantel E et al (2010) Finite element investigation of stentless pericardial aortic valves: relevance of leaflet geometry. Ann Biomed Eng 38(5):1908-1918

11. Marom G, Haj-Ali R, Rosenfeld M, Schäfers HJ, Raanani E (2013) Aortic root numeric model: correlation between intraoperative effective height and diastolic coaptation. J Thorac Cardiovasc Surg 145(1):303-304

12. Marom G, Peleg M, Halevi R, Rosenfeld M, Raanani E, Hamdan A et al (2013) Fluid-structure interaction model of aortic valve with porcine-specific collagen fiber alignment in the cusps. J Biomech Eng 135:101001-101006. doi:10.1115/1.4024824

13. Cox JL, Ad N, Myers K, Gharib M, Quijano RC (2005) Tubular heart valves: a new tissue prosthesis design—-preclinical evaluation of the $3 \mathrm{~F}$ aortic bioprosthesis. J Thoracic Cardiovasc Surg 130(2):520-527

14. Holzapfel GA, Gasser TC (2001) A viscoelastic model for fiber-reinforced composites at finite strains: continuum basis, computational aspects and applications. Comput Methods Appl Mech Eng 190:4379-4403. doi:10.1016/ S0045-7825(00)00323-6

15. Ehret AE, Itskov M (2007) A polyconvex hyperelastic model for fiber-reinforced materials in application to soft tissues. J Mater Sci 42:8853-8863. doi:10.1007/s10853-007-1812-6

16. Holzapfel GA (2004) Computational biomechanics of soft biological tissue. In: Encyclopedia of Computational Mechanics. Wiley. http://onlinelibrary.wiley.com/doi/10.1002/0470091355.ecm041/abstract

17. Reese S, Raible T, Wriggers $P$ (2001) Finite element modelling of orthotropic material behaviour in pneumatic membranes. Int J Solids Struct 38:9525-9544. doi:10.1016/S0020-7683(01)00137-8

18. Reese S (2003) Meso-macro modelling of fibre-reinforced rubber-like composites exhibiting large elastoplastic deformation. Int J Solids Struct 40:951-980. doi:10.1016/S0020-7683(02)00602-9

19. Stier B, Simon J-W, Reese S (2014) Finite element analysis of layered fiber composite structures accounting for the material's microstructure and delamination. Appl Compos Mater. doi:10.1007/s10443-013-9378-8

20. Simon J-W, Höwer D, Stier B, Reese S (2015) Meso-mechanically motivated modeling of layered fiber reinforced composites accounting for delamination. Compos Struct 122:477-487. doi:10.1016/j.compstruct.2014.12.006

21. Lechat C, Bunsell AR, Davies P (2010) Tensile and creep behaviour of polyethylene terephthalate and polyethylene naphthalate fibres. J Mater Sci 46:528-533. doi:10.1007/s10853-010-4999-x

22. Flanagan TC, Cornelissen C, Koch S, Tschoeke B, Sachweh JS, Schmitz-Rode T et al (2007) The in vitro development of autologous fibrin-based tissue-engineered heart valves through optimised dynamic conditioning. Biomaterials 28(23):3388-3397

23. Bardy J, Legrand X, Crosky A (2012) Configuration of a genetic algorithm used to optimise fibre steering in composite laminates. Compos Struct 94:2048-2056. doi:10.1016/j.compstruct.2011.12.019

24. Legrand X, Kelly D, Crosky A, Crépin D (2006) Optimisation of fibre steering in composite laminates using a genetic algorithm. Compos Struct 75:524-531. doi:10.1016/j.compstruct.2006.04.067

25. Blom AW, Abdalla MM, Gürdal Z (2010) Optimization of course locations in fiber-placed panels for general fiber angle distributions. Compos Sci Technol 70:564-570. doi:10.1016/j.compscitech.2009.12.003

26. Gliesche K, Hübner T, Orawetz H (2003) Application of the tailored fibre placement (TFP) process for a local reinforcement on an "open-hole" tension plate from carbon/epoxy laminates. Compos Sci Technol 63:81-88. doi:10.1016/S0266-3538(02)00178-1

27. Hariton I, deBotton G, Gasser TC, Holzapfel GA (2006) Stress-driven collagen fiber remodeling in arterial walls. Biomech Model Mechanobiol 6:163-175. doi:10.1007/s10237-006-0049-7

28. Annaidh AN, Bruyère K, Destrade M, Gilchrist MD, Maurini C, Otténio M et al (2012) Automated estimation of collagen fibre dispersion in the dermis and its contribution to the anisotropic behaviour of skin. Ann Biomed Eng 40(8):1666-1678

\section{Submit your manuscript to a SpringerOpen ${ }^{\circ}$ journal and benefit from:}

- Convenient online submission

- Rigorous peer review

- Immediate publication on acceptance

- Open access: articles freely available online

- High visibility within the field

- Retaining the copyright to your article

Submit your next manuscript at $\mathbf{s p r i n g e r o p e n . c o m ~}$ 